\title{
Dark energy cosmologies for codimension-two branes
}

\author{
Jan-Markus Schwindt ${ }^{1}$, Christof Wetterich ${ }^{2}$ \\ ${ }^{1}$ Institut für Physik, Universität Mainz, Staudingerweg 7, 55128 Mainz \\ E-mail: Schwindt@thep.physik.uni-mainz.de \\ ${ }^{2}$ Institut für Theoretische Physik, Universität Heidelberg, Philosophenweg 16, 69120 Heidelberg \\ E-mail: C.Wetterich@thphys.uni-heidelberg.de
}

\begin{abstract}
A six-dimensional universe with two branes in the "football-shaped" geometry leads to an almost realistic cosmology. We describe a family of exact solutions with time dependent characteristic size of internal space. After a short inflationary period the late cosmology is either of quintessence type or turns to a radiation dominated Friedmann universe where the cosmological constant appears as a free integration constant of the solution. The radiation dominated universe with relativistic fermions is analyzed in detail, including its dimensional reduction.
\end{abstract}

\section{Introduction}

The cosmological constant problem is the question why the observed curvature of spacetime is so much smaller (by 120 orders of magnitude) than the naively guessed gravitational scale. In higher dimensional theories, such as Kaluza-Klein theories or braneworlds, the total curvature $R^{(4+d)}$ can be divided into three parts: the four-dimensional (4D) curvature $R^{(4)}$ of the visible universe, the curvature $R^{(d)}$ of internal space (the bulk) and a term for the warping. The total curvature is expected to be of the order of the fundamental scale of the theory. In this context, the cosmological constant problem can be formulated in a different way: Why is only such a small part of $R^{(4+d)}$ contained in $R^{(4)}$ ?

In this context Rubakov and Shaposhnikov noted [1 that in a six-dimensional pure gravity theory $R^{(4)}$ is a free integration constant of the general solution and may have an arbitrary value, no matter how large $R^{(6)}$ is. The same is true 2 in 6D Einstein Maxwell theory where two dimensions are compactified by magnetic flux [3]. The latter model does not share the singularities appearing in the Rubakov-Shaposhnikov model and has better stability properties [2, 3, 4. Its geometry corresponds to the bulk of braneworlds (with codimension 2) which are extensively discussed today. The remaining question is then: why would a solution with very small $R^{(4)}$ be selected? 
A somewhat similar problem appears in standard four-dimensional cosmology. The total 4D curvature of the universe consists of two terms: the 3D curvature of the spatial hypersurfaces and the Hubble parameter $H=\frac{\dot{a}^{2}}{a^{2}}$ which measures the time dependence of these hypersurfaces and corresponds to the warping. Measurements of the CMB anisotropies show that the 3D curvature is almost zero, at least much smaller than the Hubble parameter. Standard cosmology implies then that this mismatch in scale between $3 \mathrm{D}$ curvature and $4 \mathrm{D}$ curvature was even much more pronounced in the past. This raises the question: Why does the 3D curvature carry only such a small part of the total $4 \mathrm{D}$ curvature? In this case a dynamical solution to the problem is known: inflation. During inflation, the 3D curvature is exponentially driven to zero, while the $4 \mathrm{D}$ curvature is essentially given by the energy density and pressure of the inflaton, which remains almost constant.

One may ask the question if the cosmological constant problem as stated above, i.e. the question why the 4D curvature carries only such a small part of the total higher dimensional curvature, may also have a dynamical solution. This could happen in two ways: The first possibility concerns solutions which approach asymptotically for large time a static and stable internal space and where the four-dimensional cosmological constant is a free integration constant of the general solution. A very small value of this constant may be favoured by the dynamics of the very early universe when the size and shape of internal space were strongly time-dependent and the stable solution with fixed small $\Lambda_{4}$ could have been asymptotically approached. Such a scenario would be the analogue of inflation as discussed above. A second possibility is that the internal space remains time-dependent even at late times. This may lead to quintessence-type [5] effective four-dimensional cosmologies where the "dark energy" density becomes small and time-dependent by virtue of a cosmic attractor solution, independently of the parameters of the model and the integration constants of the solution. We will discuss both possibilities here, but restrict ourselves to a particular six-dimensional example.

The cosmological constant problem in six dimensions has recently been discussed within the context of braneworlds [6, 7, 8, 9, 10. In this paper we present new cosmological solutions for the so-called "football shaped" model [6]. The two-dimensional internal space has axial symmetry and two equal conical singularities at the two poles, giving it the shape of an American football. It is stabilized by the magnetic flux of the six-dimensional gauge field.

It is known that this model exhibits static solutions where the $4 \mathrm{D}$ cosmological constant appears as a free integration constant [2. In this paper we demonstrate that there exists at least one cosmological solution which approaches a given static solution asymptotically. This holds for arbitrary values of the integration constants of the general static solution. Beyond earlier work we investigate the full time dependence of the volume of internal space and the $4 \mathrm{D}$ scale factor. We establish exact cosmological solutions of the $6 \mathrm{D}$ field equations.

The time-dependent size of internal space appears in four dimensions as a timedependent scalar field which has substantial influence on cosmology. The shape of the corresponding scalar potential allows for a short period of inflation, a stable ground state with or without cosmological constant, or alternatively for a cosmology with exponential 
potential quintessence. As a second difference to other work we do not postulate that the usual matter is so much constrained on the brane that it becomes "invisible" from the bulk. Instead we investigate "holographic branes" 13 where the wave functions of all particles have a normalizable tail in the bulk, or even have a maximum there. This requires the size of the bulk to be small. In consequence, the "superheavy Kaluza-Klein modes" decay early in cosmology and play no role for the radiation or matter dominated late universe.

This point of view surrounds all the recently discussed problems with codimension-two branes and the cosmology appears to be quite realistic. Indeed, we find that the energy momentum tensor of the massless fermions or gauge fields generates a usual FriedmannRobertson-Walker cosmology in the observed four-dimensional world. Together these features locate our solutions much closer to reality than the other approaches recently discussed.

The paper is organized as follows: In section 2 we present cosmological solutions of 6D Einstein-Maxwell theory with "football shaped" internal space of time-dependent size. From a four-dimensional perspective, this size appears as a scalar field $\phi$ whose potential is determined by the bulk physics. It has in general a minimum and a maximum, and we show that inflation as well as Friedmann cosmology can be obtained in this context. In section 3 we include fermionic matter, compute its energy momentum tensor and show how the radiation necessary for a Friedmann cosmology can be described in our six-dimensional context. In section 4, we present the effective four-dimensional theory, obtained by dimensional reduction. There are two massless $\mathrm{U}(1)$ gauge fields whose couplings depend on $\phi$. In section 5 , we present our conclusions.

\section{Cosmological solutions with two symmetric branes}

Six-dimensional Einstein-Maxwell theory couples the metric $g_{A B}$ to a six-dimensional photon field $A_{A}$ according to the action

$$
S=\int d^{6} x \sqrt{-g}\left\{-\frac{M_{6}^{4}}{2} R+\lambda_{6}+\frac{1}{4} F^{A B} F_{A B}\right\} .
$$

Here $g=\operatorname{det} g_{A B}, R$ is the six-dimensional curvature scalar, the mass scale $M_{6}$ can be associated with a six-dimensional Newton constant $G_{6}=\left(8 \pi M_{6}^{4}\right)^{-1}, \lambda_{6}$ is the sixdimensional cosmological constant and the gauge field strength is given by $F_{A B}=\partial_{A} A_{B}-$ $\partial_{B} A_{A}$. The field equations read

$$
\begin{gathered}
R_{A B}-\frac{1}{2} R g_{A B}=M_{6}^{-4}\left(T_{A B}^{(F)}+T_{A B}^{(M)}-\lambda_{6} g_{A B}\right), \\
\partial_{A}\left(\sqrt{-g} F^{A B}\right)=0 .
\end{gathered}
$$

The right hand side of the Einstein equation involves the energy momentum tensor which has a contribution from the (coherent) photon field

$$
T_{A B}^{(F)}=F_{A C} F_{B}^{C}-\frac{1}{4} F_{C D} F^{C D} g_{A B}
$$


and from the incoherent fluctuations of radiation or matter $T_{A B}^{(M)}$. Let us first investigate the "vacuum solutions" of the field equations where $T_{A B}^{(M)}$ is neglected.

We find a class of exact special solutions with a line element

$$
d s^{2}=\exp \left(-\frac{\phi(t)}{\bar{M}}\right)\left\{-d t^{2}+a^{2}(t) d \vec{x} d \vec{x}\right\}+\exp \left(\frac{\phi(t)}{\bar{M}}\right) r_{0}^{2}\left\{d \rho^{2}+B^{2} \sin ^{2} \rho d \theta^{2}\right\}
$$

where

$$
r_{0}^{2}=\frac{\bar{M}^{2}}{4 \pi B M_{6}^{4}} .
$$

The gauge field in this solution has only a non-vanishing component in the $\theta$-direction ${ }^{1}$,

$$
A_{\theta}=\frac{m}{2 e_{6}}(1-\cos \rho) \text {. }
$$

Here $\vec{x}$ are cartesian comoving three-dimensional coordinates, $\theta$ is a periodic angular variable $0 \leq \theta<2 \pi$ and $\rho$ is in the interval $0<\rho<\pi$. The field equations (2), (3) are obeyed provided $H(t)=\dot{a}(t) / a(t)$ (dots denote time derivatives) and $\phi(t)$ obey

$$
\begin{gathered}
H^{2}=\frac{1}{3 \bar{M}^{2}}\left(\frac{1}{2} \dot{\phi}^{2}+V(\phi)\right), \\
\ddot{\phi}+3 H \dot{\phi}+\frac{\partial V}{\partial \phi}=0
\end{gathered}
$$

with a potential

$$
V(\phi)=\bar{M}^{4}\left\{\frac{\lambda_{6}}{M_{6}^{4} \bar{M}^{2}} e^{-\frac{\phi}{M}}-4 \pi B \frac{M_{6}^{4}}{\bar{M}^{4}} e^{-\frac{2 \phi}{M}}+2 \pi^{2} m^{2} \frac{M_{6}^{4}}{e_{6}^{2} \bar{M}^{6}} e^{-\frac{3 \phi}{M}}\right\} .
$$

We recognize in eqs. (8), (9) the well-known system of four-dimensional cosmological field equations for a homogeneous isotropic universe with a scalar field $\phi$. For our ansatz (51) we have introduced a Weyl scaling factor multiplying the 4D metric in order to guarantee that the effective $4 \mathrm{D}$ Newton's constant $8 \pi G=\bar{M}^{-2}$ is independent of time. The appearance of $\phi$ in exponential form is required if $\phi$ is normalized such that its kinetic term takes the standard form. [11, 12

Besides the six-dimensional parameters $M_{6}, \lambda_{6}$ and $e_{6}$ our family of solutions contains several free integration constants: $\bar{M}$ and $B$ as well as the "initial values" of $\phi$ and $\dot{\phi}$ at $t=0$ are continuous integration constants, whereas $m$ is integer. We will see that the integration constant $B$ plays a particular role: we will find a family of realistic cosmologies where the effective four-dimensional cosmological constant $\lambda_{4}$ depends continuously on $B$. Therefore $\lambda_{4}$ appears as an integration constant of the solution rather than being a parameter which is calculable from the six-dimensional couplings.

Before discussing the properties of $V(\phi)$ and the associated cosmological solutions, we should understand the geometrical properties of the ansatz. For fixed $t$ and $\vec{x}$ the internal space spanned by $\rho, \theta$ has singularities at $\rho=0, \pi$ if $B \neq 1$. In this case it has

\footnotetext{
${ }^{1}$ In a coordinate patch around $\rho=\pi$, one should use $A_{\theta}=\frac{m}{2 e_{6}}(-1-\cos \rho)$.
} 
an "American football shaped" geometry with conic singularities at $\rho=0, \pi$ where the deficit angle is

$$
\Delta=2 \pi(1-B)
$$

One can interpret this geometry in terms of two branes sitting at $\rho=0$ and $\rho=\pi$. The brane tension can be directly inferred from the bulk geometry. With a procedure analogous to the one described in [13], one finds that the energy momentum tensor $T_{A B}^{(B)}$ of the brane has a $\delta$-distribution like form with non-vanishing components only in the $\mu, \nu$ (4D) direction,

$$
\left(T^{(B)}\right)_{\mu}^{\nu}=\frac{B-1}{B r_{0}^{2} e^{\phi / \bar{M}}} M_{6}^{4}\left(\frac{\delta(\rho)}{\rho}+\frac{\delta(\rho-\pi)}{\pi-\rho}\right) \delta_{\mu}^{\nu} .
$$

The tension is given by

$$
\mu=-\int_{0}^{\epsilon} d \rho d \theta \sqrt{g_{\rho \rho} g_{\theta \theta}}\left(T^{(B)}\right)_{t}^{t}=2 \pi(1-B) M_{6}^{4}
$$

This is independent of $\phi$ and corresponds to the energy density of the brane per fourdimensional volume ${ }^{2}$ which is therefore time-independent. However, we consider the brane tension here as a dynamical quantity, similar in spirit to the mass of a black hole in the four-dimensional world. In principle, the tension could change with time, although it does not for the special solutions considered here. Also similar to a black hole, the brane never needs to be considered explicitly: the singularities can be inferred from the bulk geometry by a type of "holographic principle" 13. Only the six-dimensional field equations need to be solved.

For the special case $B=1$ the internal space becomes a sphere and no branes are present. The isometry group is now enhanced to $\mathrm{SO}(3)$. For this case the cosmological solutions of the 6D Einstein-Maxwell theory have been extensively discussed in [11. Actually, the local solutions with $B \neq 1$ can be mapped to the previously known local solutions for $B=1$ by the variable change $\theta^{\prime}=B \theta$, accompanied by a rescaling $e_{6}^{\prime}=B e_{6}$. (Of course, the periodicity of $\theta^{\prime}$ differs from $2 \pi$ if $B \neq 1$.) By multiplying the gauge coupling in the formulae of [11] by a factor of $B$ they can be taken over directly to the case of a football shaped geometry.

The gauge field configuration (17) corresponds to a "monopole" provided $m$ is an integer. Indeed, once additional charged fields, e.g. fermions, are introduced, the sixdimensional gauge coupling $e_{6}$ (which has dimension mass $^{-1}$ ) becomes an additional free parameter of the action. In addition to the overall mass scale, that we may take as $M_{6}$, our model is therefore characterized by two dimensionless quantities $\lambda_{6} / M_{6}^{6}$ and $e_{6}^{2} M_{6}^{2}$. For the case $B=1$ the monopole configuration is actually consistent with the spherical symmetry if rotations are accompanied by suitable gauge transformations 3 .

\footnotetext{
${ }^{2}$ Since the brane tension does not appear as an energy density in the effective $4 \mathrm{D}$ theory and is thus a manifestly six-dimensional quantity, the $4 \mathrm{D}$ volume is computed here with the $6 \mathrm{D}$ metric $g_{\mu \nu}$, not with the effective $4 \mathrm{D}$ metric $\tilde{g}_{\mu \nu}=g_{\mu \nu} e^{\phi / \bar{M}}$. Otherwise the tension would indeed depend on $\phi$ and hence on time.
} 


\section{$2.1 \quad$ Inflationary Universe}

The character of the possible cosmological solutions depends on the shape of the potential $V(\phi)$. We observe that $V$ depends on the integration constant $B$ which is associated with the deficit angle. In terms of the variable $x=\left(M_{6}^{2} / \bar{M}^{2}\right) \exp (-\phi / \bar{M})$ and the dimensionless parameter combinations $\tilde{\lambda}=\lambda_{6} /\left(4 \pi M_{6}^{6}\right)$ and $\tilde{\mu}=\pi m^{2} /\left(2 e_{6}^{2} M_{6}^{2}\right)$, the potential reads

$$
V(x)=4 \pi \bar{M}^{4}\left(\tilde{\lambda} x-B x^{2}+\tilde{\mu} x^{3}\right) .
$$

For $\tilde{\mu}>0$ and $B^{2}>3 \tilde{\mu} \tilde{\lambda}$ it exhibits a maximum at

$$
x_{\max }=\frac{B}{3 \tilde{\mu}}\left(1-\sqrt{1-\frac{3 \tilde{\mu} \tilde{\lambda}}{B^{2}}}\right)
$$

and a minimum at

$$
x_{\min }=\frac{B}{3 \tilde{\mu}}\left(1+\sqrt{1-\frac{3 \tilde{\mu} \tilde{\lambda}}{B^{2}}}\right) .
$$

The maximum also exists for $\tilde{\mu}=0$ at $x_{\max }=\tilde{\lambda} / 2 B$. At the maximum $V_{\max }=V\left(x_{\max }\right)$ is always positive and we find a solution with an exponential expansion of the scale factor

$$
a(t)=a_{0} \exp \left(H_{\max } t\right), \quad H_{\max }^{2}=\frac{V_{\max }}{3 \bar{M}^{2}}
$$

and time independent $\phi=\phi_{\max }$. This solution is unstable, and solutions starting in the vicinity of $\phi_{\max }$ may be associated with the inflationary universe [11. However, the curvature of the potential around the maximum is of the order of the Hubble parameter,

$$
\begin{gathered}
\mu_{\max }^{2}=\left.\frac{\partial^{2} V}{\partial \phi^{2}}\right|_{\phi_{\max }}=-c H_{\max }^{2}, \\
c=18\left(1-\frac{1}{2-\frac{B}{\lambda} x_{\max }}\right),
\end{gathered}
$$

such that a small deviation $\delta \phi=\phi-\phi_{\max }$ grows rapidly on a time scale set by $H_{\max }^{-1}$,

$$
\begin{gathered}
\delta \phi=\delta \phi_{0} \exp \left(\gamma H_{\max } t\right) \\
\gamma^{2}+3 \gamma-c=0 .
\end{gathered}
$$

A sufficient number of e-foldings during inflation would require an extremely tiny $\delta \phi_{0}$. One concludes [11] that the present model exhibits the de Sitter solution suitable for inflation but does not have the quantitative properties for inflation to last sufficiently long. 


\subsection{Late cosmology with cosmological constant}

The minimum exists only for $m \neq 0$ - the role of the magnetic field is precisely to lead to a stable "ground state". Near $\phi_{\min }$ the cosmology depends crucially on the value of the effective four-dimensional cosmological constant $\lambda_{4}=V_{\min }=V\left(x_{\min }\right)$. For $\lambda_{4}=0$ we find a static ground state with four-dimensional Poincare invariance, i.e. $\phi=\phi_{\min }, H=0$. For very small $\lambda_{4} / \bar{M}^{4}$ an interesting cosmological solution starts with $\phi\left(t_{0}\right)$ near $\phi_{\text {max }}$ in an inflationary phase, with $\phi$ then moving towards $\phi_{\min }$ and finally performing damped oscillations around the minimum. Adding the incoherent fluctuations of radiation and matter, this corresponds to a quite realistic cosmology!

For arbitrary values of the six-dimensional parameters $\lambda_{6}, M_{6}$ and $e_{6}$ and arbitrary $m \neq 0$ there exists always a suitable choice of $B=\bar{B}$ such that $\lambda_{4}=0$,

$$
\bar{B}^{2}=4 \tilde{\mu} \tilde{\lambda}=\frac{m^{2} \lambda_{6}}{2 e_{6}^{2} M_{6}^{8}} .
$$

There is therefore always a solution that approaches the Friedmann cosmology asymptotically. Indeed, the cosmological solution can be described in this case by a transition from a de Sitter universe during the inflationary period to a flat ground state. For $B=\bar{B}$ and with $y=B x /(2 \tilde{\lambda})$ the potential takes the simple form

$$
V=\frac{2 \bar{B}^{3} \bar{M}^{4} M_{6}^{4} e_{6}^{4}}{\pi m^{2}} y(1-y)^{2}
$$

This agrees with [1] for $\bar{B}=1$. Whereas the condition $\bar{B}=1$ amounts to a tuning of the six-dimensional parameters, we have now a whole family of solutions where $B$ is an integration constant such that the flat space condition $B=\bar{B}$ can be obeyed for arbitrary values of the six-dimensional parameters. Furthermore there are also other solutions with $B \neq \bar{B}$ that lead asymptotically to an expansion characterized by a nonzero cosmological constant $\lambda_{4}$. For the particular fine tuned choice $B-\bar{B} \approx 10^{-120}$ one would obtain a realistic cosmology with $\lambda_{4}=\left(2 \times 10^{-3} \mathrm{eV}\right)^{4}$, except for the too short inflation $(c=9 / 2$, $\gamma=3(\sqrt{3}-1) / 2)$.

\subsection{Quintessence Cosmology}

Another type of "quintessence cosmology" arises if $\phi$ moves away from the inflationary solution near $\phi_{\max }$ into the direction of large $\phi$, i.e. $\phi>\phi_{\max }$. Without radiation or matter the asymptotic behavior for large $t$ would correspond in this case to

$$
H=2 t^{-1}, \quad \phi=2 \bar{M} \ln \frac{t}{\sqrt{10} M_{6}^{2} \lambda_{6}^{-1 / 2}} .
$$

In presence of radiation or matter, the same solution is approached. The fraction of homogeneous "dark energy" converges to 1 ,

$$
\Omega_{h}=\frac{V+\frac{1}{2} \dot{\phi}^{2}}{3 \bar{M}^{2} H^{2}} \rightarrow 1,
$$


already at early times (i.e. not much later than a possible entropy production at the end of inflation) and there would be no radiation or matter dominated era. At late times the energy density of the scalar field decreases according to

$$
V+\frac{1}{2} \dot{\phi}^{2} \propto t^{-2}
$$

while matter and radiation decrease as

$$
\rho_{m} \propto t^{-6}, \quad \rho_{r} \propto t^{-8}
$$

respectively. The reason for this unwanted behavior is the precise form of the exponential factor in the dominant term of the potential for large $\phi$, namely $\exp \left(-\frac{\phi}{M}\right)$. For potentials proportional to $\exp \left(-\frac{\phi}{k M}\right)$ with $k<\frac{1}{2}$ exist attractor solutions where the dark energy density adapts to the incoherent part of the energy density and approaches

$$
\Omega_{h} \rightarrow k^{2} n,
$$

where $n=4$ for radiation and $n=3$ for matter domination. Coefficients $k<\frac{1}{2}$ may be expected to be obtained for the corresponding scalar potential in suitable models with higher codimensions.

Another problem of these "quintessence" solutions is that, as we will demonstrate in section 4, the present simple model would lead to a substantial time dependence of the couplings in the effective four-dimensional theory. This is a direct consequence of the fact that the action does not exhibit a dilatation symmetry. Nevertheless we mention the interesting feature that, independently of all six-dimensional parameters and the values of $m$ or $B$, the asymptotic value of the dark energy density vanishes. The potential $V(\phi)$ always approaches zero for $\phi \rightarrow \infty$, and not some positive or negative constant. In this restricted sense our model solves both cosmological constant problems, i.e. the asymptotic vanishing of the energy density for $t \rightarrow \infty$ and the explanation of a tiny nonzero number $V / \bar{M}^{4}$. In our model the tiny number is simply a consequence of the huge age of the universe. Finally we note that the "quintessence" cosmology can also be obtained for $m=0$, i.e. in the pure six-dimensional Einstein theory without a photon field.

\section{Radiation dominated universe}

Next we want to include radiation and matter into the specific cosmologies described above. We work in the context of "holographic branes" [13. where all particles correspond to modes with a normalizable tail in the bulk. Then the matter content is fully specified in terms of the normalizable modes of the six-dimensional bulk geometry. Again no particular specification of physics on the brane is needed since a type of "holographic principle" allows for an extrapolation of the bulk properties to the brane. We consider the incoherent fluctuations of two massless charged six-dimensional Weyl fermions $\Psi$ with opposite abelian charges $\pm q$ and equal six-dimensional helicity ${ }^{3}$. Each of these fermion

\footnotetext{
${ }^{3}$ The existence of charged particles restricts the allowed monopole numbers such that $q m$ is an integer.
} 
fields can be harmonically expanded,

$$
\Psi(x, \theta, \rho)=\sum \psi_{\ln }(x) \zeta_{\ln }(\theta, \rho),
$$

where $\zeta_{l n}$ are eigenfunctions of the internal Dirac operator and each mode $\zeta$ can be split into a $\theta$ and a $\rho$ dependent part,

$$
\zeta_{l n}=e^{i n \theta} \chi_{l n}(\rho)
$$

After dimensional reduction the eigenvalues of the internal Dirac operator are seen in four dimensions as mass terms. For most modes the mass is huge of the order of the compactification scale $M_{c} \sim r_{0}^{-1} \exp (-\phi / \bar{M})$. At low energies only the massless or very light modes are relevant. Unless the existence of light fermions is required by symmetry arguments, their appearance would be a pure coincidence and would require some finetuning of parameters. We therefore assume that the light 4D fermions are precisely the unpaired chiral modes in the above expansion. These modes are required to be massless in the case of unbroken symmetry by virtue of a nonvanishing chirality index [14. In the football shaped model unpaired chiral modes exist only for fermions that are charged with respect to the gauge field $A$ [13]. For $B=1$ they form an irreducible $|q m|$-dimensional representation of the larger isometry group $\mathrm{SO}(3)$, whereas for $B \neq 1$ the degeneracy may be broken. For the energy momentum tensor of these massless modes we expect a relativistic equation of state. If the $\mathrm{U}(1)$-symmetries are spontaneously broken, the fermions typically acquire masses of the order of the symmetry breaking scale. This would lead to a late time cosmology with nonrelativistic matter. We neglect spontaneous symmetry breaking here and stick to a radiation dominated universe.

Since we are interested in possible late time cosmologies, we will assume the energy density of these fermions to be small as compared to the other variables which determine the structure of internal space: the 6D cosmological constant and the magnetic flux. The fermions can therefore be considered as a perturbation, with only a tiny backreaction on the shape of internal space. Furthermore we assume that the characteristic time scale of the cosmological dynamics is large as compared to $M_{c}^{-1}$. To a good approximation, the internal wave functions of the fermions will then be given by the zero modes of the internal Dirac operator on a static football shaped background with deficit angle $\Delta$. Only the normalization constant $N_{l n}(t)$ may depend on time due to the possible time dependence of the volume of internal space.

In the case of the football shaped model, the zero modes can be computed explicitly. The internal Dirac equation is

$$
i \Gamma^{\alpha} D_{\alpha} \zeta(\rho, \theta)=0
$$

Here $D_{\alpha}$ is the covariant derivative containing the spin connection and the gauge coupling, the index $\alpha$ runs over $\theta$ and $\rho$. One gets

$$
D_{\theta}=\partial_{\theta}+\frac{1}{2} i \tau_{3}(1-B \cos \rho)-i e_{6} q A_{\theta}, \quad D_{\rho}=\partial_{\rho}
$$

The two internal gamma matrices are defined by $\Gamma^{\alpha}=e^{\alpha}{ }_{a} \tau^{a}$, where $e^{\alpha}{ }_{a}$ is the inverse 
internal vielbein and $\tau^{1}$ and $\tau^{2}$ are the first and second Pauli matrices. This yields

$$
i \Gamma^{\alpha} D_{\alpha}=\left(\begin{array}{cc}
0 & -\frac{e^{-i \theta}}{r_{0} e^{\phi / 2 M}}\left(\partial_{\rho}-\frac{i}{B \sin \rho} D_{\theta}^{-}\right) \\
\frac{e^{i \theta}}{r_{0} e^{\phi / 2 M}}\left(\partial_{\rho}+\frac{i}{B \sin \rho} D_{\theta}^{+}\right) & 0
\end{array}\right),
$$

where $D_{\theta}^{ \pm}$is $D_{\theta}$ with $\tau_{3}$ replaced by \pm 1 . Consider first the spinor with charge $+q$. The solutions to eq. (31) with $\tau_{3}$ eigenvalue +1 are

$$
\zeta_{0 n}^{+}(\rho, \theta)=N(t)(\sin \rho)^{-\frac{1}{2}+B^{-1}\left(q m-\left(n+\frac{1}{2}\right)\right)}(1-\cos \rho)^{B^{-1}\left(n+\frac{1}{2}-\frac{q m}{2}\right)}\left(\begin{array}{c}
e^{i n \theta} \\
0
\end{array}\right),
$$

where the requirement of normalizability [15, 13] constrains $n$ within the limits

$$
-\frac{B+1}{2}<n<q m+\frac{B-1}{2} .
$$

The solutions to eq. (31) with $\tau_{3}$ eigenvalue -1 are similar except for some sign changes. The normalization conditions require now

$$
q m-\frac{B-1}{2}<n<\frac{B+1}{2} .
$$

If $B \leq 1$, only one of the two conditions (35), (36) can be fulfilled, depending on the sign of $m$. For the spinor with charge $-q$ the procedure is completely analogous. We observe that to each normalizable mode contained in the spinor with charge $+q$ corresponds a normalizable mode in the spinor with charge $-q$ with opposite sign of $n$ and opposite $\tau_{3}$ eigenvalue.

We have to compute the six-dimensional energy momentum tensor of these modes. Since they are massless, we expect a relativistic equation of state, $p / \rho=1 / 3$, where $p$ is the pressure in the effective four-dimensional world, and $\rho$ is the corresponding effective energy density. The energy momentum tensor of the fermions is given by the expectation value $^{4}$

$$
T_{B}^{A}=\left\langle\frac{1}{2} i \bar{\Psi} \gamma^{A} D_{B} \Psi+\text { h.c. }\right\rangle .
$$

We assume that the different fermion modes do not mix and have arbitrary phases with respect to each other, so that all expectation values of the type $\left\langle\psi_{i}^{\dagger} \psi_{j}\right\rangle,\left\langle\bar{\psi}_{i} \psi_{j}\right\rangle,\left\langle\psi_{i}^{\dagger} \partial_{\mu} \psi_{j}\right\rangle$ and $\left\langle\bar{\psi}_{i} \partial_{\mu} \psi_{j}\right\rangle$ vanish for different modes, $i \neq j$. (Here $\psi_{i}$ corresponds to $\psi_{l n}$ in the expansion (29).) Then we can compute the energy momentum tensor for each mode separately. We therefore take $\Psi(x, \theta, \rho)=\psi(x) \zeta(\theta, \rho)$, where $\zeta=e^{i n \theta} \chi(\rho)$ is one particular zero mode. Furthermore we assume that with each mode also the corresponding antiparticle (with opposite four-dimensional handedness, opposite "winding number" $n$ and opposite charge $q$ with respect to gauge field) is excited with the same density, so that no net charges appear. For each particle contained in one of the six-dimensional Weyl spinors,

\footnotetext{
${ }^{4}$ Usually an energy momentum tensor contains also a piece $L \delta_{B}^{A}$, where $L$ is the Lagrangian density, but this vanishes here, since $L=0$ for solutions of the Dirac equation.
} 
the antiparticle is contained in the other six-dimensional Weyl spinor. Every operator $\gamma^{A} D_{A}$ is a tensor product (or a sum of tensor products) of an operator acting on the $4 \mathrm{D}$ part $\psi(x)$ and an operator acting on the internal part $\zeta(\rho, \theta)$ of the fermions. We will refer to these as the $4 \mathrm{D}$ and the $2 \mathrm{D}$ part of the operator. In the absence of warping, a possible choice for the $6 \mathrm{D}$ gamma matrices is

$$
\gamma^{\mu}=e^{\phi / 2 \bar{M}} \tilde{\gamma}^{\mu} \otimes 1, \quad \gamma^{\theta}=e^{\theta}{ }_{a} \tilde{\Gamma} \otimes \tau^{a}, \quad \gamma^{\rho}=e^{\rho}{ }_{a} \tilde{\Gamma} \otimes \tau^{a}
$$

Here a tilde denotes the $4 \mathrm{D}$ part of an operator and $\tilde{\Gamma}$ is the $4 \mathrm{D} \gamma^{5}$ matrix. The index $a$ runs from 1 to 2 . (In the presence of warping, the warp factor would have to be included into the definition of $\gamma^{\mu}$.) The covariant derivative $D_{\mu}$ contains only the $4 \mathrm{D}$ part $D_{\mu}=\tilde{D}_{\mu}$, i.e. the $2 \mathrm{D}$ part is just the identity. (This would not be true in the presence of warping.) On the other hand, $D_{\theta}$ and $D_{\rho}$ have only a $2 \mathrm{D}$ part apart from the factor $\tilde{\Gamma}$.

We assume that the distribution of the fermions is homogeneous and isotropic in threedimensional space. This forbids any components containing 3D spatial indices except for diagonal ones, $T_{(i)}^{(i)}$. The effective $4 \mathrm{D}$ Dirac equation implies

$$
\bar{\psi}(x) \tilde{\gamma}^{\mu} \tilde{D}_{\mu} \psi(x)=0
$$

where a tilde again denotes a four-dimensional operator. From isotropy then follows (no summation over $(i)$ )

$$
\bar{\psi}(x) \tilde{\gamma}^{(i)} \tilde{D}_{(i)} \psi(x)=-\frac{1}{3} \bar{\psi}(x) \tilde{\gamma}^{t} \tilde{D}_{t} \psi(x) .
$$

In order to connect this to the $6 \mathrm{D}$ picture, we compute the $6 \mathrm{D}$ energy momentum tensor. For the diagonal $(\mu \mu)$ components one obtains

$$
T_{(\mu)}^{(\mu)}=i e^{\phi / 2 \bar{M}}\left\langle\bar{\psi}(x) \tilde{\gamma}^{(\mu)} \tilde{D}_{(\mu)} \psi(x)\right\rangle|\chi(\rho)|^{2} .
$$

Combined with eq.(40) this yields the relativistic equation of state $p / \rho=T^{(i)}{ }_{(i)} /\left(-T^{t}{ }_{t}\right)=$ $1 / 3$. Integrating out the internal dimensions, the effective $4 \mathrm{D}$ energy momentum tensor reads (with an appropriate normalization of $\chi$ )

$$
T_{(\mu), e f f}^{(\mu)}=i\left\langle\bar{\psi}(x) \tilde{\gamma}^{(\mu)} \tilde{D}_{(\mu)} \psi(x)\right\rangle
$$

This is precisely what one would expect for a massless fermion.

All other components of the 6D energy momentum tensor are zero. Most terms vanish because $\bar{\psi}(x) \tilde{\Gamma} \psi(x)$ is zero for a chiral fermion. This term occurs in $T^{\theta}{ }_{\theta}, T^{\theta}{ }_{\rho}, T^{\rho}{ }_{\theta}$ and $T^{\rho}{ }_{\rho}$, since the $4 \mathrm{D}$ part of $\gamma^{\alpha} D_{\beta}$ is just $\tilde{\Gamma}$. Therefore these components all vanish. It remains to be shown that the remaining off-diagonal components are also zero. We have

$$
\begin{aligned}
T_{\rho}^{t} & =\left\langle\frac{1}{2} i \bar{\Psi} \gamma^{t} D_{\rho} \Psi+\text { h.c. }\right\rangle=\left\langle\frac{1}{2} i e^{\phi / 2 \bar{M}}\left(\bar{\psi} \tilde{\gamma}^{t} \psi\right)\left(\chi^{\dagger} \partial_{\rho} \chi\right)+\text { h.c. }\right\rangle \\
& =\left\langle\frac{1}{2} i e^{\phi / 2 \bar{M}}\left(a^{-1}(t) \psi^{\dagger} \psi\right)\left(\chi^{\dagger} \partial_{\rho} \chi\right)+\text { h.c. }\right\rangle .
\end{aligned}
$$


It follows from the structure of the internal Dirac equation that $\chi$ is a real function of $\rho$ times a constant phase (in eq.(34) this phase was chosen to be 1 ). Therefore $T^{t}{ }_{\rho}$ is purely imaginary and cancelled by the hermitian conjugate. Next

$$
T^{\rho}{ }_{t}=\left\langle\frac{1}{2} i \bar{\Psi} \gamma^{\rho} \tilde{D}_{t} \Psi+\text { h.c. }\right\rangle=0
$$

because of chirality since the $4 \mathrm{D}$ part $\bar{\psi} \tilde{\Gamma} \tilde{D}_{t} \psi$ is zero. A similar argument implies $T^{\theta}{ }_{t}=0$. An interesting role is played by the $t \theta$ component,

$$
\begin{aligned}
{T^{t}}_{\theta} & =\left\langle\frac{1}{2} i \bar{\Psi} \gamma^{t} D_{\theta} \Psi+\text { h.c. }\right\rangle \\
& =\left\langle\frac{1}{2} i e^{\phi / 2 \bar{M}} a^{-1}(t)\left(\psi^{\dagger} \psi\right) \times \zeta^{\dagger}\left(\partial_{\theta}+\frac{1}{2} i \tau_{3}(1-B \cos \rho)-i e_{6} q A_{\theta}\right) \zeta+h . c .\right\rangle \\
& =-e^{\phi / 2 \bar{M}} a^{-1}(t)|\chi(\rho)|^{2}\left(n \pm \frac{1}{2}(1-B \cos \rho)-e_{6} q A_{\theta}\right)\left\langle\psi^{\dagger} \psi\right\rangle .
\end{aligned}
$$

This is real and does not vanish automatically. A net charge would indeed lead to a nonvanishing $T^{t}{ }_{\theta}$. (Such a component would certainly, through Einstein's equations, force one of the off-diagonal metric components to become nonzero, since $G^{t}{ }^{t}$ would identically vanish for a diagonal metric.) However we assume here that for each particle the corresponding antiparticle is also present with equal density. This has opposite $n$, opposite $\tau_{3}$ eigenvalue and opposite charge $q$ with respect to the $6 \mathrm{D}$ gauge field. Its $T^{t}{ }_{\theta}$ component has therefore the same absolute value but the opposite sign, and the terms cancel in the total energy momentum tensor.

The energy momentum tensor can now be inserted into the six-dimensional field equation (2). Besides the contribution from the massless fermions the (late time) energy momentum $T_{A B}^{(M)}$ will also contain a similar relativistic contribution from the two massless four-dimensional gauge fields, the photons. One observes that the "internal equations" (for $A, B$ internal indices) are not altered. This is reasonable since a change of the shape of internal space corresponds to the excitation of superheavy modes which should not be affected by a small expectation value of the fluctuations. The field equation (9) for $\phi$ (corresponding here to a variable volume of internal space) receives no source term on the r.h.s. The only modification concerns the equation for the Hubble parameter $H$ which receives an additional contribution from the energy density $\rho=-\tilde{T}_{t}^{t}$. Averaging over internal space this replaces eq. (18) by the familiar equations

$$
H^{2}=\frac{1}{3 \bar{M}^{2}}\left(\frac{1}{2} \dot{\phi}^{2}+V(\phi)+\rho\right), \quad \dot{\rho}+4 \rho=0 .
$$

For the late cosmology with cosmological constant the value of $\phi$ settles at the minimum of $V$, and therefore $\dot{\phi} \rightarrow 0, V(\phi) \rightarrow V_{0}=\lambda$. Hence we find a cosmology with radiation and a cosmological constant.

We conclude that our scenario has many features required for a realistic cosmology. Starting with a short inflationary period (if initially $\phi$ is close to $\phi_{\max }$ ), internal space reaches a stable size and shape (if $\phi<\phi_{\max }$ ), serving as a the ground state of the 
model. The universe becomes radiation dominated and expands with the usual Friedmann behavior.

We will see below how the cosmology with the inclusion of the energy momentum tensor for the massless modes can simply be described by an effective four-dimensional theory. We close the six-dimensional discussion with the remark that the massive modes contained in the six-dimensional fields can be included in a straightforward manner. For these modes the internal Dirac operator leads to a mixing of four-dimensional modes with opposite chirality and induces a mass of characteristic size $r_{0}^{-1} \exp (-\phi / M)$. For stable particles this mass dominates their contribution to the energy momentum tensor at late times. However, the massive particles are expected to decay early in the cosmological evolution and therefore play no role during the late cosmology of the radiation dominated era.

\section{Dimensional Reduction}

For late cosmology we can concentrate on the massless (or very light) modes. Then cosmology can be described within an effective four-dimensional framework. Indeed, in the zero mode ansatz, where all massive modes are neglected, the internal space may be integrated out. For the above system with relativistic fermions one obtains the effective four-dimensional Lagrangian

$$
\begin{aligned}
L^{(4)}= & -\frac{\bar{M}^{2}}{2} R+\frac{Z_{1}(\phi)}{4} F_{\mu \nu}^{(1)} F^{\mu \nu(1)}+\frac{Z_{2}(\phi)}{4} F_{\mu \nu}^{(2)} F^{\mu \nu(2)} \\
& +i \sum_{j} \bar{\psi}_{j} \gamma^{\mu}\left(\partial_{\mu}-i Q_{j}^{(1)} \bar{e}_{1} A_{\mu}^{(1)}-i Q_{j}^{(2)} \bar{e}_{2} A_{\mu}^{(2)}\right) \psi_{j}+\frac{1}{2} \partial_{\mu} \phi \partial^{\mu} \phi+V(\phi) .
\end{aligned}
$$

Here the 4D metric $g_{\mu \nu}$ is defined in the Weyl scaled form of eq.(15), with 6D line element $d s^{2}=\exp (-\phi / \bar{M}) g_{\mu \nu} d x^{\mu} d x^{\nu}+\ldots$ It determines the $4 \mathrm{D}$ Ricci scalar $R=R^{(4)}$ and is used to lower indices. The scalar potential $V(\phi)$ is given by eq.(10). The gauge field $A_{\mu}^{(1)}$ is just the dimensionally reduced form of the $6 \mathrm{D}$ gauge field $A_{\mu}$, while $A_{\mu}^{(2)}$ arises from the internal $\mathrm{U}(1)$ symmetry and is proportional to the zero mode of the $6 \mathrm{D}$ metric components $g_{\mu \theta}$. The abelian field strength $F_{\mu \nu}^{(i)}$ is defined as usual and the massless spinors $\psi_{j}$ are covariantly coupled to the gauge fields according to their abelian charges $Q_{j}^{(i)}$. We define the bare four-dimensional gauge couplings $\bar{e}_{1}$ and $\bar{e}_{2}$ such that $Z_{1}(0)=Z_{2}(0)=1$. The renormalized gauge couplings $e_{1,2}$ obey then

$$
e_{1(2)}=\frac{\bar{e}_{1(2)}}{\sqrt{Z_{1(2)}}} .
$$

We will find

$$
Z_{1}=e^{\phi / \bar{M}}, \quad Z_{2}=e^{2 \phi / \bar{M}}
$$

and conclude that the renormalized gauge couplings depend on the value of the scalar field $\phi$. In cosmologies where $\phi$ changes with time, one encounters a time variation of the 
"fundamental constants". In principle, this is a highly interesting effect which is genuine for quintessence cosmology [16, 17]. In view of the severe restrictions from observation this variation must be very small, however. Of course, if $\phi$ settles to a fixed value for the "late cosmology with a cosmological constant" discussed above, the time variation of the gauge couplings becomes unobservable. Except for the possible time variation of the gauge couplings we find that the effective four-dimensional action (47) describes a standard four-dimensional theory for massless spinors, gauge fields and graviton plus a scalar "cosmon" field.

We want to compute the $\phi$ dependence of $Z_{1}$ and $Z_{2}$. Therefore we integrate the relevant terms in the $6 \mathrm{D}$ action. The $6 \mathrm{D}$ gauge field $A_{\mu}(x, \rho, \theta)$ can be harmonically expanded,

$$
A_{\mu}(x, \rho, \theta)=\sum \tilde{A}_{\mu}^{(l m)}(x) Y^{(l m)}(\rho, \theta),
$$

where $Y^{(l m)}$ are eigenfunctions of the internal Laplacian. In the special case of $B=1$, these are just the spherical harmonics. For the zero mode we write $\tilde{A}_{\mu}^{(1)}(x)$ and omit the indices $(\mathrm{lm})$. If needed for the purpose of a clear distinction we mark four-dimensional quantities by a tilde, e.g. $g_{\mu \nu}^{(6)}=e^{-\phi / \bar{M}} \tilde{g}_{\mu \nu}$. As mentioned before, the indices of fourdimensional fields are raised and lowered with $\tilde{g}^{\mu \nu}$ and $\tilde{g}_{\mu \nu}$. The terms in the action containing $\tilde{A}_{\mu}^{(1)}$ are

$$
\begin{aligned}
S_{1} & =\frac{1}{4} \int d^{4} x d \rho d \theta(-g)^{1 / 2} F_{\mu \nu} F^{\mu \nu} \\
& =\frac{1}{4} \int d^{4} x d \rho d \theta(-\tilde{g})^{1 / 2} e^{-\phi / \bar{M}} r_{0}^{2} B \sin \rho \\
& \times e^{2 \phi / \bar{M}} \tilde{g}^{\mu \sigma} \tilde{g}^{\nu \rho}\left(\partial_{\mu} \tilde{A}_{\nu}^{(1)}-\partial_{\nu} \tilde{A}_{\mu}^{(1)}\right)\left(\partial_{\sigma} \tilde{A}_{\rho}^{(1)}-\partial_{\rho} \tilde{A}_{\sigma}^{(1)}\right) Y^{2} \\
& =\frac{1}{4} \int d^{4} x(-\tilde{g})^{1 / 2} e^{\phi / \bar{M}} F_{\mu \nu}^{(1)} F^{\mu \nu(1)} \int d \cos \rho d \theta B r_{0}^{2} Y^{2}
\end{aligned}
$$

and

$$
\begin{aligned}
S_{2} & =i \int d^{4} x d \rho d \theta(-g)^{1 / 2} \bar{\Psi} \gamma^{\mu}\left(\partial_{\mu}-i q e_{6} A_{\mu}\right) \Psi \\
& =i \int d^{4} x d \rho d \theta(-\tilde{g})^{1 / 2} e^{-\phi / \bar{M}} r_{0}^{2} B \sin \rho \\
& \times \sum_{j} \zeta_{j}^{\dagger}(\rho, \theta) \zeta_{j}(\rho, \theta) \bar{\psi}_{j}(x) \gamma^{m} \tilde{e}_{m}^{\mu} e^{\phi / 2 \bar{M}}\left(\partial_{\mu}-i q e_{6} \tilde{A}_{\mu}^{(1)} Y(\rho, \theta)\right) \psi_{j}(x) .
\end{aligned}
$$

The proper normalization of the kinetic term of the fermions requires

$$
\int d \cos \rho d \theta e^{-\phi / 2 \bar{M}} B r_{0}^{2} \zeta_{j}^{\dagger} \zeta_{j}=1
$$

for each $j$. Since in $S_{2}$ the $\phi$ dependence is absorbed by this normalization, $Y$ has to be independent of $\phi$ in order to lead to a $\phi$-independent $\bar{e}_{1}$,

$$
Q_{j}^{(1)} \bar{e}_{1}=e^{-\phi / 2 \bar{M}} B r_{0}^{2} q e_{6} \int d \cos \rho d \theta \zeta_{j}^{\dagger} \zeta_{j} Y
$$


The normalization $Z_{1}(0)=1$ yields

$$
\int d \cos \rho d \theta B r_{0}^{2} Y^{2}=1
$$

and the last line of eq.(51) implies eq.(49). In consequence, the renormalized gauge coupling decreases with increasing $\phi$,

$$
e_{1}=\bar{e}_{1} e^{-\phi / 2 \bar{M}}
$$

Next we consider the gauge field corresponding to the invariance of the metric under translations in $\theta$. The Killing vector which generates the $\mathrm{U}(1)$ symmetry has only a non-vanishing $\theta$ component,

$$
K^{\theta}=\text { const }, \quad K_{\theta}=\text { const } \times \sin ^{2} \rho e^{\phi / \bar{M}} .
$$

The gauge field $A_{\mu}^{(2)}$ is defined by

$$
g_{\mu \theta}(x, \rho, \theta)=\text { const } \times A_{\mu}^{(2)}(x) K_{\theta}(t, \rho, \theta)=C A_{\mu}^{(2)}(x) \sin ^{2} \rho e^{\phi / \bar{M}}
$$

where the constant $C$ is to be determined. The kinetic term for the second gauge field is contained in the six-dimensional Ricci scalar,

$$
R_{\text {gauge }}=-\frac{1}{4} g^{\mu \rho} g^{\nu \sigma} g^{\theta \theta}\left(\partial_{\mu} g_{\nu \theta}-\partial_{\nu} g_{\mu \theta}\right)\left(\partial_{\rho} g_{\sigma \theta}-\partial_{\sigma} g_{\rho \theta}\right)
$$

Integrating out the internal dimensions for the corresponding part of the zero mode action we get

$$
\begin{aligned}
S_{3} & =-\frac{M_{6}^{4}}{2} \int d^{4} x d \rho d \theta \sqrt{-g} R_{\text {gauge }} \\
& =\frac{1}{4} \int d^{4} x \sqrt{-\tilde{g}} F_{\mu \nu}^{(2)} F^{\mu \nu(2)} e^{2 \phi / \bar{M}} C^{2} \frac{\pi M_{6}^{4}}{B} \int_{0}^{\pi} d \rho \sin ^{3} \rho
\end{aligned}
$$

and the normalization $Z_{2}(0)=1$ yields

$$
C^{2}=\frac{3 B}{4 \pi M_{6}^{4}}
$$

The coupling to fermions is given by the term

$$
S_{4}=i \int d^{4} x d \rho d \theta \sqrt{-g} \bar{\Psi} \gamma^{m} e_{m}^{\theta} D_{\theta} \Psi
$$

Taking $e_{m}^{\theta}=-e_{m}^{\mu} g^{\theta \theta} g_{\theta \mu}$ and making use of the normalization condition (53) the integration yields

$$
S_{4}=\sum_{j} \int d^{4} x \sqrt{-\tilde{g}} \bar{\psi}_{j} \tilde{\gamma}^{\mu} A_{\mu}^{(2)} \psi_{j}\left(\mp i\left(n+\frac{1}{2}\right)\right) \frac{C}{B^{2} r_{0}^{2}} .
$$

The charges are

$$
Q_{j}^{(2)}= \pm\left(n+\frac{1}{2}\right)
$$

where the plus is for left-handed and the minus for right-handed modes. Plugging in the expressions for $r_{0}$ and $C$, we find eq.(49) and the effective gauge coupling

$$
e_{2}=2 \sqrt{3 \pi} \frac{M_{6}^{2}}{\bar{M}^{2}} e^{-\phi / \bar{M}}
$$

This completes our calculation of the effective 4D action. 


\section{Conclusions}

The six-dimensional Einstein-Maxwell theory can lead to an almost realistic cosmology. We have discussed solutions with a time-varying size of an "internal space" characterized by two branes and a "football-shaped" geometry. With a relativistic energy momentum tensor induced by the incoherent fluctuations of six-dimensional fermions, gauge fields and metric components we have shown how a Friedmann-Robertson-Walker cosmology arises at late time. The size of internal space plays the role of a four-dimensional scalar field $\phi$ which is closely associated to dark energy, either in the form of a cosmological constant $\left(\phi<\phi_{\max }\right)$ or in the form of an exponential potential quintessence $\left(\phi>\phi_{\max }\right)$.

Apart from the many realistic features of our model there remain also considerable problems. The cosmological constant problem is solved for the quintessence solutions with $\phi>\phi_{\max }$ in the sense that the ratio of the potential energy $V$ over $\bar{M}^{4}$ decreases asymptotically to zero for late time, independently of the values of the six-dimensional parameters or the choice of the integration constants of the solution. However, in this case the effective 4D gauge couplings are time-dependent beyond the observational limits, and the matter density decreases too fast. The second problem is probably rather easy to solve and the transition to a realistic radiation dominated cosmology may be achieved in suitably modified higher dimensional models. This only requires a different value of $k$ (or a different prefactor for the kinetic term for $\phi$ ). Stabilizing the running of the gauge couplings is a challenge - it may be met in higher dimensional theories with anomalous dilatation symmetry and a fixed point behavior for the running couplings [18.

For the solutions with $\phi<\phi_{\max }$, the couplings approach constant values, but here the cosmological constant problem reappears in a somewhat different form. For the present solutions an integration constant needs to be tuned. One may ask if a dynamical adjustment mechanism could favor a small value of $\lambda_{4}$, similar to the mechanism of inflation which makes the 3D curvature small compared to the 4D curvature. Furthermore, the period of inflation is in both cases much too short - again, this may be different in other higher dimensional models as already demonstrated in 11, 12. And finally, the particle spectrum (i.e. the spectrum of zero modes) is not rich enough to account for the complicated fermion spectrum that we observe.

The present model may be rendered more realistic by suitable generalizations. The necessity of tuning an integration constant for the cosmological constant for $\phi<\phi_{\text {max }}$ may be due to our limitation to only a very special kind of geometry, namely the "football shaped" one. A more general ansatz would start with an arbitrary geometry in the very early universe. It is an interesting speculation that in the much larger space of general solutions consistent with the symmetries (i.e. the U(1) isometry, gauge symmetry and invariance under four-dimensional diffeomorphisms) a solution with very small 4D cosmological constant is preferred for dynamical reasons, and is therefore approached for a large class of initial conditions. We will discuss this issue in a separate paper.

A sufficient inflation may be induced by adding higher curvature terms [12, which are very likely to be present as corrections in the effective action since they are generated by quantum fluctuations. These higher curvature terms may also be responsible for the compactification of internal space, instead of the gauge field. Such a stabilization mecha- 
nism becomes important for branes with codimension higher than two, where the simple monopole configuration does not exist.

A more realistic fermion spectrum is obtained when one generalizes the monopole configuration of the ground state to a non-abelian gauge field. A gauge group SO(12) instead of $\mathrm{U}(1)$ leads to a remarkably realistic fermion spectrum [19, with three generations of quarks and leptons with the appropriate quantum numbers. In this model one finds even most of the features required for the observed mass hierarchies and small mixings between the fermions.

Many interesting speculations and discussions invoke unusual features of gravity which could be generated from higher dimensional physics and modify the late cosmology. We believe that it is timely to construct realistic higher dimensional cosmologies that lead to the more "standard" type of a late universe with radiation, matter and dark energy. This will establish benchmarks for judging the naturalness of the more drastic modifications.

\section{References}

[1] V. A. Rubakov and M. E. Shaposhnikov, Phys. Lett. B 125, 139 (1983)

[2] C. Wetterich, Nucl. Phys. B 255, 480 (1985)

[3] S. Randjbar-Daemi, A. Salam and J. Strathdee, Nucl.Phys. B 214, 491 (1983)

[4] G. V. Lavrelashvili and P. G. Tinyakov, Sov. J. Nucl. Phys. 41, 172 (1985)

[5] C. Wetterich, Nucl.Phys. B 302, 668 (1988); B. Ratra and P. J. E. Peebles, Phys. Rev. D 37, 3406 (1988)

[6] S. M. Carroll and M. M. Guica, hep-th/0302067; I. Navarro, JCAP 0309, 004 (2003)

[7] J. M. Cline, J. Descheneau, M. Giovannini and J. Vinet, JHEP 0306, 048 (2003); J. Vinet and J. M. Cline, Phys. Rev. D 70, 083514 (2004)

[8] Y. Aghababaie, C. P. Burgess, S. L. Parameswaran and F. Quevedo, Nucl. Phys. B 680, 389 (2004)

[9] H. P. Nilles, A. Papazoglou and G. Tasinato, Nucl. Phys. B 677, 405 (2004)

[10] I. Navarro and J. Santiago, hep-th/0411250

[11] C. Wetterich, Nucl.Phys. B 252, 309 (1985)

[12] Q. Shafi and C. Wetterich, Phys.Lett.B 129, 387 (1983)

[13] J. Schwindt and C. Wetterich, Phys. Lett. B 578, 409 (2004)

[14] C. Wetterich, Nucl. Phys. B 223, 109 (1983); E. Witten, Proc. Shelter Island Conf. (1983) 
[15] C. Wetterich, Nucl. Phys. B 253, 366 (1985)

[16] C. Wetterich, Nucl. Phys. B 302, 645 (1988)

[17] G. R. Dvali and M. Zaldarriaga, Phys. Rev. Lett. 88, 091303 (2002)

[18] C. Wetterich, Phys. Lett. B 561, 10 (2003)

[19] C. Wetterich, Nucl.Phys. B 244, 359 (1984); Nucl.Phys. B 260, 402 (1985); Nucl.Phys. B 261, 461 (1985); Nucl.Phys. B 279, 711 (1987) 